\title{
Component-trees and multivalued images: Structural properties
}

\author{
Nicolas Passat, Benoît Naegel
}

\begin{abstract}
Component-trees model the structure of grey-level images by considering their binary level-sets obtained from successive thresholdings. They also enable to define anti-extensive filtering procedures for such images. In order to extend this image processing approach to any (grey-level or multivalued) images, both the notion of component-tree, and its associated filtering framework, have to be generalised. In this article we first deal with the generalisation of the component-tree structure. We define a new data structure, the component-graph, which extends the notion of component-tree to images taking their values in any (partially or totally) ordered set. The component-graphs are declined in three variants, of increasing richness and size, whose structural properties are studied.
\end{abstract}

Index Terms-Mathematical morphology, component-tree, multivalued images, anti-extensive filtering, component-graph.

\section{INTRODUCTION}

$\mathbf{T}$ HE component-tree is a data structure which models some characteristics of grey-level images by considering their binary level-sets obtained from successive thresholding operations. Component-trees are particularly well-suited for the design of methods devoted to process or analyse greylevel images, based on hypotheses related to the topology (connectedness) and the specific intensity (local extrema) of structures of interest. Based on these properties, componenttrees have been involved in several image processing applications, especially for filtering and segmentation.

The success of component-trees in the field of grey-level image processing, together with the increasing need for applications involving multivalued images, justify their extension to the case of such images, which do not take their values in totally ordered sets, but in any (partially or totally) ordered ones. In particular, this work takes place in the context of the extension of mathematical morphology to multivalued images.

After a preliminary study of the relations between component-trees and multivalued images from a methodological point of view [1], a generalisation of component-trees to such images has been initiated in [2]. The present work develops this framework. In particular, this article deals with data structure issues (algorithmic issues will be considered in a further article). We describe a new data structure, the component-graph, which extends the notion of component-tree to images taking values in any ordered set. The componentgraphs are declined in three variants of increasing richness and size. There structural properties are studied, in particular under various hypotheses related to frequent image value spaces.

Nicolas Passat and Benoît Naegel are with the Université de Strasbourg, LSIIT UMR CNRS 7005, Strasbourg, France (passat@unistra.fr, b.naegel@unistra.fr).
This article is organised as follows. Section II describes the context of this work. Section III provides notations. Section IV gives a formal definition of the classical notion of componenttree. Sections V-VII constitute the contribution of the article. Section V defines the notion of component-graph. Section VI establishes the structural links between different variants of this notion. Section VII studies the influence of specific image value spaces, thus leading to structural simplifications. Section VIII summarises the principal properties of componentgraphs. Section IX concludes the article.

\section{RELATED WORKS}

\section{A. Component-trees}

Initially proposed in the field of statistics [3], [4], the component-tree (also known as dendrone [5], [6], confinement tree [7] or max-tree [8]) has been (re)defined in the framework of mathematical morphology (see, e.g., [9]-Chapter 7, or [10]) and involved in the development of morphological operators [11], [8].

From a methodological point of view, some efforts have been conducted to enable the efficient computation of component-trees [8], [12], [13]. From an applicative point of view, component-trees have been involved in the development of several image processing and analysis techniques. Most of them are devoted to filtering or segmentation [8], [14], [15], [16], [17]. Other applications have also been considered, for instance, image registration [7], [18], image retrieval [19], [20], image classification [21], interactive visualisation [22], multithresholding [23] or document binarisation [24].

In the field of filtering and segmentation, the proposed methods have been designed to detect some structures of interest by using information modelled by attributes [11], [25], and stored at each node of the tree. These attributes are chosen according to hypotheses related to the applicative context. The subtree obtained by pruning the component-tree of the image, with respect to these attributes, can then be used to reconstruct a binary (segmentation) or grey-level (filtering) result.

\section{B. Mathematical morphology and multivalued images}

Mathematical morphology has been first defined on binary images, and then on grey-level ones (see [9] for a recent state of the art on mathematical morphology). Its extension to multivalued (e.g., colour, multispectral, label) images is an important task, motivated by potential applications in multiple areas. Several contributions have been devoted to this specific purpose (a whole state of the art is beyond the scope of this article; see, e.g., [26] for a recent survey). 
In general, the spaces in which such images take their values are not canonically equipped with total orders (by opposition to the case of grey-level images), but with partial ones. Several strategies have been considered to deal with this issue. Except in few works (see, e.g., [27]), they intend to split these value spaces into several totally ordered ones (marginal processing), or to define ad hoc total order relations on them (vectorial processing), with several variants [28], [29], [30], [31], [32]. These approaches present the advantage of embedding multivalued images into simpler frameworks which authorise to process them similarly to grey-level ones, enabling in particular to reduce the algorithmic complexity induced by partially ordered sets. Unfortunately, they also potentially bias the information intrinsically carried by these -more complex but richer-partially ordered value spaces.

In the present work, we deal with the general case of any (partially or totally) ordered value spaces, without attempting to modify the order, then providing a contribution to the extension of mathematical morphology to multivalued images, and more especially to the one of connected filtering (see also [33], [34] for other recent contributions on this topic).

\section{NOTATIONS}

The inclusion (resp. strict inclusion) relation on sets is noted $\subseteq$ (resp. $\subset$ ). The cardinality of a set $X$ is noted $|X|$. The power set of a set $X$ is noted $2^{X}$. If $\mathcal{P} \subseteq 2^{X}$ is a partition of $X$, we write $X=\bigsqcup \mathcal{P}$.

A function $F$ from a set $X$ to a set $Y$ is noted $F: X \rightarrow Y$, and the set of all the functions from $X$ to $Y$ is noted $Y^{X}$. If $X^{\prime} \subseteq X$ and $Y^{\prime} \subseteq Y$, we note $F\left(X^{\prime}\right)=\left\{F(x) \mid x \in X^{\prime}\right\}$ and $F^{-1}\left(Y^{\prime}\right)=\left\{x \in X \mid F(x) \in Y^{\prime}\right\}$. If $F$ is a bijection, we also note $F^{-1}: Y \rightarrow X$ its associated inverse function.

Let $\frown$ be a (binary) relation on a set $X$. The restriction of $\frown$ to a subset $Y \subseteq X$ will generally still be noted $\frown$ (except if a new notation is introduced).

We say that $\frown$ is an equivalence relation if $\frown$ is reflexive, transitive and symmetric. For any $x \in X$, the equivalence class of $x$ with respect to $\frown$ is noted $[x]_{\curvearrowright}$. The set of all these equivalence classes is noted $X / \frown$.

We say that $\frown$ is an order relation (and that $(X, \frown)$ is an ordered set) if $\frown$ is reflexive, transitive and antisymmetric. Moreover, we say that $\frown$ is a total (resp. partial) order relation (and that $(X, \frown)$ is a totally (resp. partially) ordered set), if $\frown$ is total (resp. partial) (i.e., if $\forall x, y \in X,(x \frown y) \vee(y \frown x)$ (resp. if $\exists x, y \in X,(x \nmid y) \wedge(y \nmid x))$ ).

For any symbol further used to denote an order relation $(\subseteq$, $\leq, \unlhd$, etc.), the inverse symbol ( $\supseteq, \geq$, $\unrhd$, etc.) denotes the associated dual order, while the symbol without lower bar $(\subset$, $<, \triangleleft$, etc.) denotes the associated strict order.

The Hasse diagram of an ordered set $(X, \leqslant)$ is the couple $(X, \prec)$ where $\prec$ is the cover relation associated to $\leqslant$, defined for all $x, y \in X$ by $x \prec y$ iff $x<y$ and there is no $z \in X$ such that $x<z<y$.

If $(X, \leqslant)$ is an ordered set and $x \in X$, we note $x^{\uparrow}=$ $\{y \in X \mid y \geqslant x\}$ and $x^{\downarrow}=\{y \in X \mid y \leqslant x\}$, namely the sets of the elements greater and lower than $x$, respectively. If $Y \subseteq X$, the set of all the maximal and minimal elements of
$X$ are noted $\nabla^{\leqslant} Y$ and $\Delta^{\leqslant} Y$, respectively. The supremum and the infimum of $Y$ are noted (when they exist) $V^{\leqslant} Y$ and $\Lambda^{\leqslant} Y$, respectively (we will note $\bigcup$ and $\cap$ for $\bigvee^{\subseteq}$ and $\Lambda^{\subseteq}$, respectively). The maximum and the minimum of $Y$ are noted (when they exist) $\gamma^{\leqslant} Y$ and $\Lambda^{\leqslant} Y$, respectively. If $Y$ is defined as $\{x \mid p(x)\}$ where $p$ is a Boolean predicate, we will sometimes note $\Upsilon_{p(x)}^{\leqslant} x$, instead of $\Upsilon^{\leqslant} Y$; the same remark holds for $\Lambda^{\leqslant}, V^{\leqslant}, \Lambda \leqslant, \bigcup, \cap, \sqcup$.

(Note that the symbol $\leq$ will be used to denote two distinct orders: the standard order on $\mathbb{Z}$ and the pointwise order on functions; the context of use enables however to unambiguously associate the correct semantics to each occurrence of the symbol.)

\section{COMPONENT-TREES}

\section{A. Connectivity}

Intuitively (and informally), the notion of connectivity on a set $\Omega$ allows to decide whether it is possible to go from a point (or a subset) of $\Omega$ to another one while always remaining in $\Omega$. If this property is verified, we say that $\Omega$ is connected. Several (similar, and sometimes equivalent [35]) ways can be considered to define connectivity: from the standard notions of topology [36], [37]; from the notions of paths in digital/discrete spaces [38], [39], [40]; or even by morphological definitions of connectivity [41], [42], [43], [16].

A connectivity on $\Omega$ can be defined by a function $\mathcal{C}: 2^{\Omega} \rightarrow$ $2^{2^{\Omega}}$ which provides, for any $X \subseteq \Omega$ the set of all the connected sets included in $X$. The maximal elements of the ordered set $(\mathcal{C}(X), \subseteq)$ are called the connected components of $X$, and the set of all the connected components of $X$ (namely $\nabla^{\subseteq} \mathcal{C}(X)$ ) is noted $\mathcal{C}[X]$.

In the present work, we are mainly interested by the following three properties of connectivity:

(P1) If $X \subseteq \Omega$, then the set $\mathcal{C}[X]$ is a partition of $X$.

(P2) If $X \subseteq Y \subseteq \Omega$, then for any $A \in \mathcal{C}[X]$, there exists a unique $B \in \mathcal{C}[Y]$ such that $A \subseteq B$.

(P3) If $X \subseteq Y \subseteq \Omega, A \in \mathcal{C}[Y]$ and $A \subseteq X$, then $A \in \mathcal{C}[X]$.

Broadly speaking, Property (P1) guarantees the completeness and non-redundancy of the decomposition of $X$ into connected components; Property (P2) guarantees the hierarchical organisation of connected components in the power set lattice of $\Omega$; and Property (P3) guarantees the persistence of connected components in this lattice.

In the sequel, we consider any connectivity on $\Omega$ (satisfying, in particular, Properties (P1)-(P3)), provided that $\Omega$ is connected for this connectivity (i.e., $\mathcal{C}[\Omega]=\{\Omega\}$ ).

In the illustrations of Sections IV-VII, the sets $\Omega$ will be finite subsets of $\mathbb{R}^{2}$ equipped with the usual arc-based connectivity.

\section{B. Images}

Let $\Omega$ be a nonempty finite set. Let $V$ be a nonempty finite set equipped with an order relation $\leqslant$. We assume that $(V, \leqslant)$ admits a minimum, noted $\perp$. An image is a function $I: \Omega \rightarrow$ $V$. The sets $\Omega$ and $V$ are called the support and the value space of $I$, respectively. For any $x \in \Omega, I(x) \in V$ is the 
value of $I$ at $x$. Without loss of generality, we assume that $I^{-1}(\{\perp\}) \neq \emptyset$. If $(V, \leqslant)$ is a totally (resp. partially) ordered set, we say that $I$ is a grey-level (resp. a multivalued) image.

Let $X \subseteq \Omega$ and $v \in V$. The thresholding function $\lambda_{v}$ is defined by

$$
\mid \begin{array}{ll}
\lambda_{v}: V^{\Omega} & \rightarrow 2^{\Omega} \\
& I \quad \mapsto\{x \in \Omega \mid I(x) \geqslant v\}
\end{array}
$$

The cylinder function $C_{(X, v)}$ is defined by

$$
\begin{aligned}
C_{(X, v)}: \Omega & \rightarrow V \\
x & \mapsto \begin{cases}v & \text { if } x \in X \\
\perp & \text { otherwise }\end{cases}
\end{aligned}
$$

An image $I: \Omega \rightarrow V$ can be decomposed into cylinder functions induced by thresholding operations and, symmetrically, $I$ can be reconstructed by composition of these cylinder functions, as

$$
I=\bigvee_{v \in V} \bigvee_{X \in \mathcal{C}\left[\lambda_{v}(I)\right]}^{\leq} C_{(X, v)}
$$

where $\leq$ is the order relation on $V^{\Omega}$ defined by

$$
(F \leq G) \Leftrightarrow(\forall x \in \Omega, F(x) \leqslant G(x))
$$

We note $\Psi$ the set of all the connected components obtained from all the thresholdings of $I$

$$
\Psi=\bigcup_{v \in V} \mathcal{C}\left[\lambda_{v}(I)\right]
$$

\section{Component-trees}

In the sequel of this section, we assume that $(V, \leqslant)$ is a totally ordered set. It derives from Properties (P1), (P2) and from the totality of $\leqslant$ that for any $X \in \Psi,\left(X^{\uparrow}, \subseteq\right)$ is a totally ordered set. Moreover, since we also have $\Omega=\gamma^{\subseteq} X^{\uparrow}$, the Hasse diagram $\mathfrak{T}$ of $(\Psi, \subseteq)$ has a tree structure (of root $\Omega$ ). This Hasse diagram is called the component-tree of $I$.

An example of component-tree is shown in Fig. 1(a-g). It illustrates the fact that $X \in \Psi$ can correspond to several connected components in distinct thresholded images $\lambda_{v}(I) \subseteq \Omega$ for successive values $v \in V$.

This remark implies that $X \in \Psi$ is intrinsically associated in $\mathfrak{T}$ to a value $m(X)$ defined by

$$
m(X)=\stackrel{\leqslant}{\bigvee}\left\{v \mid X \in \mathcal{C}\left[\lambda_{v}(I)\right]\right\}=\widehat{\curlywedge}_{x \in X}^{\leqslant} I(x)
$$

which is actually the maximal value of $V$ which generates this connected component by thresholding of $I$ (note that the second equality in Formula (6) derives from Property (P3)). This definition of $m(X)$ is justified by the reconstruction of $I$ from its component-tree. Indeed, each $X \in \Psi$ is associated to a cylinder function, and in particular to the value parameterising this function. More formally, based on Formula (3), we have

$$
I=\bigvee_{X \in \Psi}^{\leq} C_{(X, m(X))}
$$

The selection of subsets of $\Psi$ (generally based on ad hoc criteria) in component-trees $\mathfrak{T}$ can be used to develop (antiextensive) filtering procedures [8], [14]. When performing

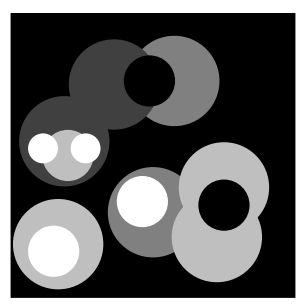

(a) $I$

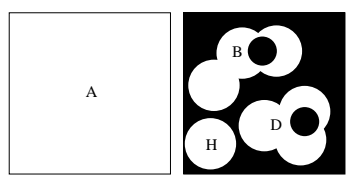

(c) $\lambda_{0}(I)$

(d) $\lambda_{1}(I)$

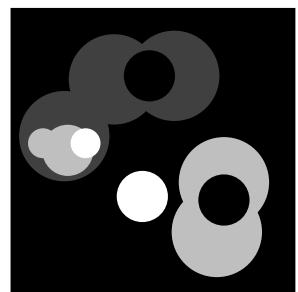

(h) Filtering $(\widehat{I})$

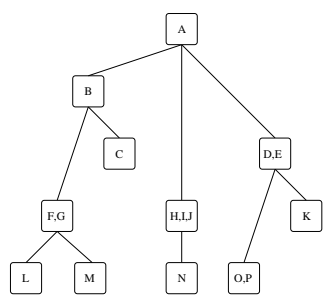

(b) $\mathfrak{T}$

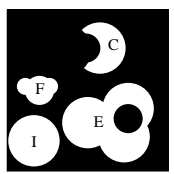

(e) $\lambda_{2}(I)$

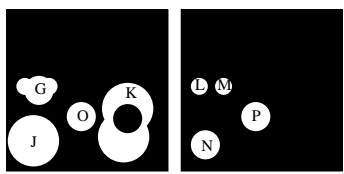

(f) $\lambda_{3}(I)$

(g) $\lambda_{4}(I)$
Fig. 1. (a) A grey-level image $I: \Omega \rightarrow V$ with $\Omega \subset \mathbb{R}^{2}$, and $V=\llbracket 0,4 \rrbracket \subset$ $\mathbb{Z}$ (from 0 , in black, to 4 , in white), equipped with the standard order relation $\leq$ on $\mathbb{Z}$. (c-g) Thresholded images $\lambda_{v}(I) \subseteq \Omega$ (in white) for $v$ varying from 0 to 4 . (b) The component-tree $\mathfrak{T}$ of $I$. The letters (A-P) in nodes correspond to the associated connected components $(\mathrm{c}-\mathrm{g})$. (h) Filtered image $\widehat{I}$ obtained from $I$ by preserving the nodes B, F, K, M and P in $\mathfrak{T}$. (i) Segmented (binary) image obtained from $I$ by substituting $\bigcup$ to $Y \leq$, and $X$ to $C_{(X, m(X))}$ in Formula (7), with the same set of nodes as in (h).

such procedures, the resulting image $\widehat{I}: \Omega \rightarrow V$ induced by $\widehat{\Psi} \subseteq \Psi$ can be defined by substituting $\widehat{\Psi}$ to $\Psi$ in Formula (7), as illustrated in Fig. 1(h,i).

\section{Purpose}

We propose to extend the concepts defined in Section IV-C to the case of multivalued images. In order to do so, it is necessary:

(i) to define a data structure generalising the notion of component-tree to such images; and

(ii) to generalise the associated filtering framework accordingly.

The item $(i)$ is considered in Sections V-VII. The item $(i i)$ will be developed in a subsequent article.

\section{COMPONENT-GRAPHS}

In this section, we assume that the relation order $\leqslant$ on $V$ can be either partial or total.

\section{A. Valued connected components}

In Formula (3), any cylinder function $C_{(X, v)}$ is generated by a couple $(X, v)$ where $X \in \mathcal{C}\left[\lambda_{v}(I)\right]$ is a connected component of the thresholded image $\lambda_{v}(I) \subseteq \Omega$ of $I$ at value $v$. In the sequel, $(X, v)$ is called a valued connected 
component. We define the set $\Theta \subseteq 2^{\Omega} \times V$ of all the valued connected components of an image $I: \Omega \rightarrow V$ as

$$
\Theta=\bigcup_{v \in V} \mathcal{C}\left[\lambda_{v}(I)\right] \times\{v\}
$$

From the order relation $\leqslant$ defined on $V$, and the inclusion relation $\subseteq$ on $2^{\Omega}$, we can define the order relation $\unlhd$ on $\Theta$ as follows

$$
\left(X_{1}, v_{1}\right) \unlhd\left(X_{2}, v_{2}\right) \Leftrightarrow\left\{\begin{array}{l}
\left(X_{1} \subset X_{2}\right) \vee \\
\left(\left(X_{1}=X_{2}\right) \wedge\left(v_{2} \leqslant v_{1}\right)\right)
\end{array}\right.
$$

This order relation on the valued connected components can be seen as the analogue of the (order) inclusion relation on the connected components. In particular, for any $\left(X_{1}, v_{1}\right),\left(X_{2}, v_{2}\right) \in \Theta$, it verifies the following properties

$$
\begin{aligned}
\left(\left(X_{1} \cap X_{2} \neq \emptyset\right)\right. & \left.\wedge\left(v_{2} \leqslant v_{1}\right)\right) \\
\left(\left(X_{1}, v_{1}\right) \unlhd\left(X_{2}, v_{2}\right)\right) & \Rightarrow\left(v_{1} \nless X_{2}\right)
\end{aligned}
$$

\section{B. Component-graphs}

In first approximation, the component-graph $\mathfrak{G}$ of an image $I: \Omega \rightarrow V$ is defined as the Hasse diagram of the ordered set $(\Theta, \unlhd)$. However, three variants of component-graphs can relevantly be considered by defining two additional subsets $\dot{\Theta}, \ddot{\Theta} \subseteq \Theta$ of valued connected components (the usefulness of which will be justified in the sequel)

$$
\begin{aligned}
& \dot{\Theta}=\bigcup_{X \in \Psi}\{X\} \times \bigvee\left\{v \mid X \in \mathcal{C}\left[\lambda_{v}(I)\right]\right\} \\
& \ddot{\Theta}=\{(\Omega, \perp)\} \cup \bigcap\left\{\Theta^{\prime} \subseteq \Theta \mid I=\bigvee_{K \in \Theta^{\prime}}^{\leq} C_{K}\right\}
\end{aligned}
$$

Broadly speaking, $\Theta$ gathers all the valued connected components induced by $I$; $\dot{\Theta}$ gathers the valued connected components of maximal values for any connected components; and $\ddot{\Theta}$ gathers the valued connected components associated to the cylinders functions which are sup/max-generators of $I$ (see Formula (3)).

We note $\triangleleft$ (resp. $\dot{\triangleleft}$, resp. $\ddot{4})$ the cover relation associated to the order relation $\unlhd$ on $\Theta$ (resp. to the restriction of $\unlhd$ to $\dot{\Theta}$, resp. to the restriction of $\unlhd$ to $\ddot{\Theta}$ ). From these definitions, we derive that

$$
\ddot{\Theta} \subseteq \dot{\Theta} \subseteq \Theta
$$

and

$$
\begin{aligned}
& \stackrel{\unlhd}{\bigvee} \Theta=\stackrel{\unlhd}{\bigvee} \dot{\Theta}=\stackrel{\unlhd}{\bigvee} \ddot{\Theta}=(\Omega, \perp) \\
& \stackrel{\unlhd}{\triangle} \Theta=\stackrel{\unlhd}{\triangle} \dot{\Theta}=\unlhd^{\unlhd} \ddot{\Theta}
\end{aligned}
$$

We then have the following definition for the three variants of component-graphs.

Definition 1 (Component-graph(s)): Let $I: \Omega \rightarrow V$ be an image. The $\Theta-\left(\right.$ resp. $\dot{\Theta}_{-}$, resp. $\left.\ddot{\Theta}-\right)$ component-graph of $I$ is the Hasse diagram $\mathfrak{G}=(\Theta, \varangle)$ (resp. $\dot{\mathfrak{G}}=(\dot{\Theta}, \dot{\mathbf{4}})$, resp. $\ddot{\mathfrak{G}}=(\ddot{\Theta}, \ddot{\triangleleft}))$ of the ordered set $(\Theta, \unlhd)$ (resp. $(\dot{\Theta}, \unlhd)$, resp. $(\ddot{\Theta}, \unlhd)$ ). For the sake of concision, the term $\Theta$-component-

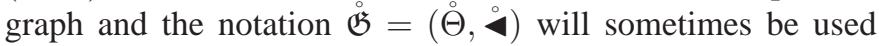

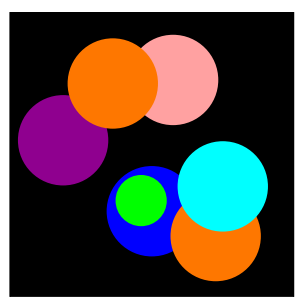

(a) $I$

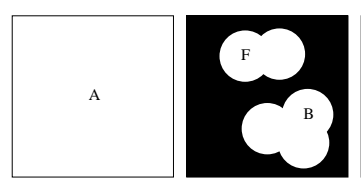

(c) $\lambda_{a}(I)$

(d) $\lambda_{b}(I)$

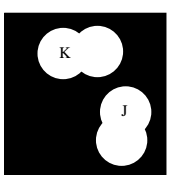

(i) $\lambda_{g}(I)$

(m) $\mathfrak{G}$ (h) $\lambda_{f}(I)$
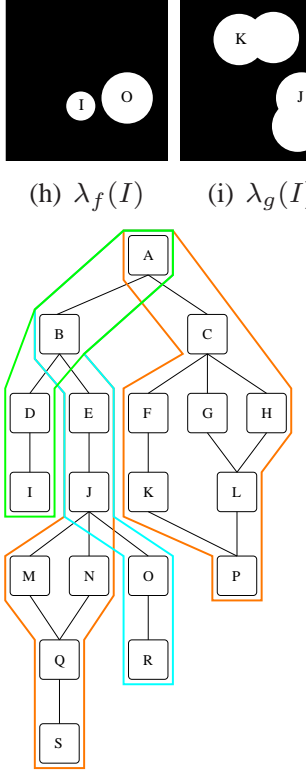

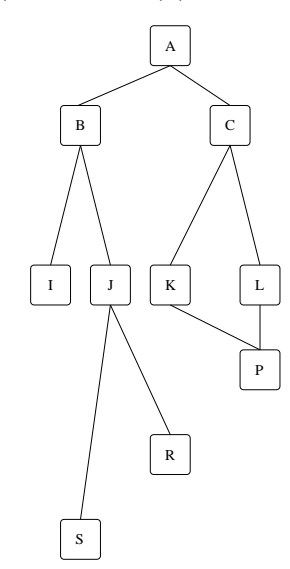

(n) $\dot{\mathfrak{G}}$

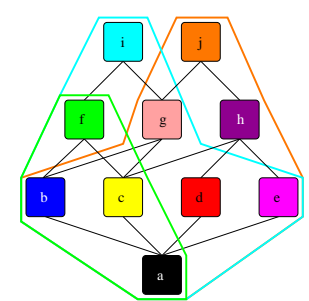

(b) $(V, \prec)$
Fig. 2. (a) A multivalued image $I: \Omega \rightarrow V$ with $\Omega \subset \mathbb{R}^{2}$, and $V=$ $\{a, b, c, d, e, f, g, h, i, j\}$. (b) The Hasse diagram of the ordered set $(V, \leqslant)$. For the sake of readability, each value of $V$ is associated to an arbitrary colour. The (Hasse diagrams of the) ordered sets $\left(f^{\downarrow}, \leqslant\right),\left(i^{\downarrow}, \leqslant\right),\left(j^{\downarrow}, \leqslant\right)$ are identified by the closed green, cyan and orange curves, respectively. (c-1) Thresholded images $\lambda_{v}(I)$ for $v \in V$. (m) The $\Theta$-component-graph of $I$. The letters (A-S) in nodes correspond to the associated connected components (c-1). For any one of the four leaves $K=(X, v) \in \Delta^{\unlhd} \Theta$, the (Hasse diagram of the) ordered set $\left(K^{\uparrow}, \unlhd\right)$ is identified by a closed curve of colour corresponding to the value $\sigma(K)=v$ (see Property 1). The comparison between these four curves and the three curves of (b) illustrates the "flattening" of $\left(K^{\uparrow}, \unlhd\right)$ by comparison to $\left(\sigma(K)^{\downarrow}, \leqslant\right)$ (see Property 3). (n) The $\Theta$ component-graph of $I$. The nodes $\mathrm{I}, \mathrm{J}, \mathrm{K}, \mathrm{L}, \mathrm{R}$ and $\mathrm{S}$, in $\mathfrak{G}$ are associated to the sets of nodes $\{\mathrm{D}, \mathrm{I}\},\{\mathrm{E}, \mathrm{J}\},\{\mathrm{F}, \mathrm{K}\},\{\mathrm{G}, \mathrm{H}, \mathrm{L}\},\{\mathrm{O}, \mathrm{R}\}$ and $\{\mathrm{M}, \mathrm{N}, \mathrm{Q}$, $\mathrm{S}\}$ in $\mathfrak{G}$, respectively (see Property 4 ). (o) The $\Theta ̈$-component-graph of $I$. The nodes $\mathrm{C}$ and $\mathrm{J}$, which are surrounded by $\{\mathrm{K}, \mathrm{L}\}$ and $\{\mathrm{R}, \mathrm{S}\}$, respectively, belong to $\dot{\Theta}$ but not to $\ddot{\Theta}$ (see Property 6).

to denote the three kinds of component-graphs. The elements of $\Theta$ are called $\Theta$-nodes (or simply, nodes); the elements of $\checkmark$ are called $\Theta$-edges (or simply, edges); $(\Omega, \perp)$ is called the

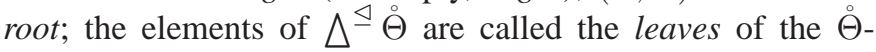
component-graph.

An example of component-graph is illustrated in Fig. 2.

From Formula (3), the reconstruction of $I$ from its valued 
connected components is given by

$$
I=\bigvee_{K \in \Theta}^{\leq} C_{K}
$$

\section{Vi. General Remarks on the Structure of COMPONENT-GRAPHS}

In this section, we study some links existing between the different structures of $\Theta$-component-graphs and the Hasse diagram $(V, \prec)$ of $(V, \leqslant)$.

\section{A. Links between $(\Theta, \triangleleft)$ and $(V, \prec)$}

The $\Theta$-component-graph of $I: \Omega \rightarrow V$ locally inherits from the structure of $(V, \prec)$. More precisely, for any leave $K=(X, v) \in \Delta^{\unlhd} \Theta$, the ordered set $\left(K^{\uparrow}, \unlhd\right)$ is "similar" to the ordered set $\left(v^{\downarrow}, \leqslant\right)$.

This similarity is first expressed by the fact that each valued connected component of $K^{\uparrow}$ can be associated to a unique value of $v^{\downarrow}$ and vice versa.

Property 1: Let $K=(X, v) \in \Delta^{\unlhd} \Theta$. The function

$$
\mid \begin{array}{ll}
\sigma: K^{\uparrow} & \rightarrow v^{\downarrow} \\
(Y, u) & \mapsto u
\end{array}
$$

is a bijection between $K^{\uparrow}$ and $v^{\downarrow}$.

Proof: The fact that $\sigma((Y, u)) \in v^{\downarrow}$ for any $(Y, u) \in K^{\uparrow}$ derives from the fact that $K \in \Delta^{\unlhd} \Theta$ (this is generally not true otherwise). The injectivity and surjectivity of $\sigma$ then derive from Properties (P1) and (P2).

This similarity is also expressed by the fact that the relation between two values of $v \downarrow$ is preserved between their associated valued connected components in $K^{\uparrow}$.

Property 2: Let $K=(X, v) \in \Delta^{\unlhd} \Theta$. The function $\sigma^{-1}$ : $v^{\downarrow} \rightarrow K^{\uparrow}$ induces a homomorphism from $\left(v^{\downarrow}, \geqslant\right)$ to $\left(K^{\uparrow}, \unlhd\right)$ : for any $K_{1}=\sigma^{-1}\left(v_{1}\right), K_{2}=\sigma^{-1}\left(v_{2}\right) \in K^{\uparrow}$, we have

$$
\left(v_{1} \geqslant v_{2}\right) \Rightarrow\left(K_{1} \unlhd K_{2}\right)
$$

Proof: The result derives from Property (P2).

In general, this homomorphism is not an isomorphism. In particular, it is possible that $\left(X_{1}, v_{1}\right) \unlhd\left(X_{2}, v_{2}\right)$ while $v_{2} \nless v_{1}$. This property derives from the definition of $\unlhd$ (Formula (9)), in which $\subseteq$ is considered prioritarily to $\leqslant$.

From a theoretical point of view, the ordered set $\left(K^{\uparrow}, \unlhd\right)$ is then richer than $\left(v^{\downarrow}, \leqslant\right)$. (In other words, the cardinality of $\unlhd$ is higher than the cardinality of $\leqslant$.) However, from a practical point of view $\left(K^{\uparrow}, \varangle\right)$ is (most of the time) less rich than $\left(v^{\downarrow}, \prec\right)$.

Property 3: Let $K=(X, v) \in \Delta^{\unlhd} \Theta$. Let $v_{1}, v_{2} \in v^{\downarrow}$ and $K_{1}=\sigma^{-1}\left(v_{1}\right), K_{2}=\sigma^{-1}\left(v_{2}\right) \in K^{\uparrow}$. Let us suppose that $K_{1} \unlhd K_{2}$ while $v_{2} \nless v_{1}$. Let $v_{3} \in v^{\downarrow}$ and $K_{3}=\sigma^{-1}\left(v_{3}\right) \in$ $K^{\uparrow}$. We have

$$
\begin{aligned}
& \left(v_{3} \prec v_{1}\right) \Rightarrow\left(\left(v_{3} \prec v_{2}\right) \Rightarrow K_{1} \nless K_{3}\right) \\
& \left(v_{2} \prec v_{3}\right) \Rightarrow\left(\left(v_{1} \prec v_{3}\right) \Rightarrow K_{3} \nless K_{2}\right)
\end{aligned}
$$

Proof: If $v_{3} \prec v_{1}, v_{2}$, we have $v_{3} \leqslant v_{1}, v_{2}$ and then $K_{1}, K_{2} \unlhd K_{3}$. By hypothesis, we then have $K_{1} \unlhd K_{2} \unlhd K_{3}$. By definition of $\triangleleft$ it comes $K_{1} \downarrow K_{3}$, and Formula (20) then holds. A similar reasoning can be applied for Formula (21).

Broadly speaking, the appearance of one edge in $\left(K^{\uparrow}, \boldsymbol{4}\right)$ results in the disappearance of $k$ edges $(k \in \mathbb{N})$, by comparison to the edges of $\left(v^{\downarrow}, \prec\right)$. In particular, the different parts of $(\Theta, \triangleleft)$ located above each leaf, will often be "flattened", by comparison to $\left(v^{\downarrow}, \prec\right)$, thus reducing the size of the associated data structure. This phenomenon is exemplified in Fig. 2(a,b, $m)$.

\section{B. Links between $(\dot{\Theta}, \dot{\hookrightarrow})$ and $(\Theta, \varangle)$}

The nodes of $(\Theta, \triangleleft)$ which are preserved in $(\dot{\Theta}, \dot{\triangleleft})$ are those which are maximal elements with respect to $\unlhd$, for a given connected component (see Formula (12)).

Let $\sim_{\AA}$ be the equivalence relation on $\stackrel{\odot}{\Theta}$ defined by

$$
\left(\left(X_{1}, v_{1}\right) \sim_{\AA}\left(X_{2}, v_{2}\right)\right) \Leftrightarrow\left(X_{1}=X_{2}\right)
$$

(These relations gather in their equivalence classes the valued connected components which correspond to similar connected components.) Note that for any node $K \in \dot{\Theta}$, we have

$$
[K]_{\sim_{\dot{\theta}}}=\stackrel{\unlhd}{\unlhd}[K]_{\sim_{\theta}}
$$

Broadly speaking, any set of nodes $[K]_{\sim_{\theta}}$ of $(\Theta, \triangleleft)$ leads to a set of nodes $[K]_{\sim_{\dot{\theta}}}$ of $(\dot{\Theta}, \dot{\hookrightarrow})$.

The links between the edges of $(\Theta, \triangleleft)$ and those of $(\dot{\Theta}, \dot{\hookrightarrow})$ are characterised as follows.

Property 4: Let $\left[K_{1}\right]_{\sim_{\dot{\theta}}}$, $\left[K_{2}\right]_{\sim_{\dot{\theta}}}$ be two distinct equivalence classes of $\sim_{\dot{\theta}}$. Then the following two assertions are equivalent

$$
\begin{gathered}
\exists K_{1}^{\prime} \in\left[K_{1}\right]_{\sim_{\theta}}, K_{2}^{\prime} \in\left[K_{2}\right]_{\sim_{\theta}}, K_{1}^{\prime} \triangleleft K_{2}^{\prime} \\
\forall K_{1}^{\prime \prime} \in\left[K_{1}\right]_{\sim_{\dot{\theta}}}, K_{2}^{\prime \prime} \in\left[K_{2}\right]_{\sim_{\dot{\theta}}}, K_{1}^{\prime \prime} \triangleleft K_{2}^{\prime \prime}
\end{gathered}
$$

Proof: The equivalence between Formulae (24) and (25) derives from the non-existence of $X \in \Psi$ such that $X_{1} \subset$ $X \subset X_{2}$, with $K_{1}=\left(X_{1}, v_{1}\right), K_{2}=\left(X_{2}, v_{2}\right)$.

Broadly speaking, all the edges between two nodes of $(\Theta, \varangle)$ associated to a same connected component disappear in $(\dot{\Theta}, \dot{\mathbf{4}})$, while any edge between two nodes of $(\Theta, \mathbf{4})$ associated to distinct connected components leads to edges between all pairs of nodes of $(\dot{\Theta}, \dot{\hookrightarrow})$ respectively associated to these two distinct connected components. These links between $\Theta$ - and $\dot{\Theta}$-component-graphs are exemplified in Fig. 2(m,n).

In this example, for any $K \in \dot{\Theta}$, we have $[K]_{\sim_{\dot{\theta}}}=\{K\}$. However, this is not necessarily true in general. Nevertheless, since the existence of an edge between $K_{1}$ and $K_{2}$ in $(\dot{\Theta}, \dot{\hookrightarrow})$ implies the existence of a similar edge between any $K_{1}^{\prime}$ and $K_{2}^{\prime}$ in $\left[K_{1}\right]_{\sim_{\dot{\theta}}}$ and $\left[K_{2}\right]_{\sim_{\dot{\theta}}}$, respectively, we can unambiguously extend the relation $\unlhd$ (and then $\dot{\triangleleft}$ ) from $\dot{\Theta}$ to $\dot{\Theta} / \sim_{\dot{\theta}}$ as follows

$$
\left(\left[K_{1}\right]_{\sim_{\dot{\theta}}} \unlhd\left[K_{2}\right]_{\sim_{\dot{\theta}}}\right) \Leftrightarrow\left(K_{1} \unlhd K_{2}\right)
$$

This enables to model $(\dot{\Theta}, \dot{\hookrightarrow})$ in a more compact fashion, by considering its equivalence classes for $\sim_{\dot{\theta}}$ instead of its nodes. This model is, moreover, directly linked to $\Psi$, as illustrated in Fig. 3. 


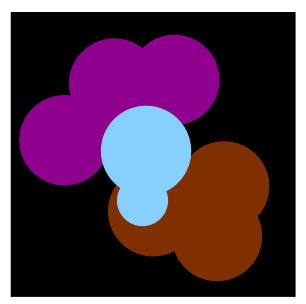

(a) $I$

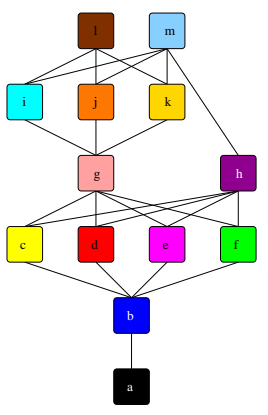

(d) $(V, \prec)$

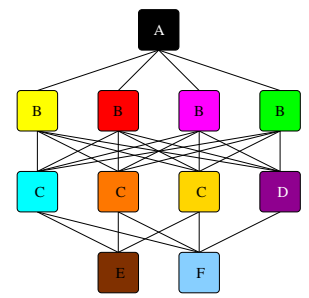

(b) $(\dot{\Theta}, \dot{4})$

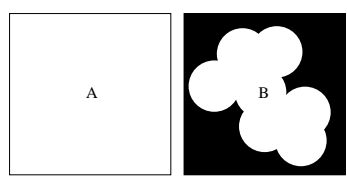

(e) $\lambda_{a}(I)$

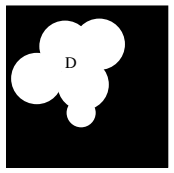

(h) $\lambda_{h}(I)$ (f) $\lambda_{f}(I)$

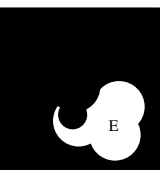

(i) $\lambda_{l}(I)$

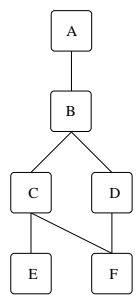

(c) $\left(\dot{\Theta} / \sim_{\dot{\theta}}, \dot{<}\right)$

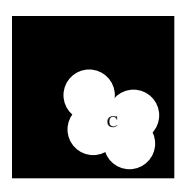

(g) $\lambda_{k}(I)$

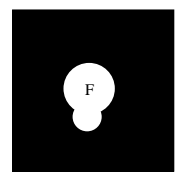

(j) $\lambda_{m}(I)$
Fig. 3. (a) A multivalued image $I: \Omega \rightarrow V$ with $\Omega \subset \mathbb{R}^{2}$, and $V=\{a, b, c, d, e, f, g, h, i, j, k, l, m\}$. (d) The Hasse diagram of the ordered set $(V, \leqslant)$. For the sake of readability, each value of $V$ is associated to an arbitrary colour. (e-j) Thresholded images $\lambda_{v}(I)$ for $v \in V$ : (e) $\lambda_{a}(I)$; (f) $\lambda_{b}(I)=\lambda_{c}(I)=\lambda_{d}(I)=\lambda_{e}(I)=\lambda_{f}(I)$; (g) $\lambda_{g}(I)=\lambda_{i}(I)=$ $\lambda_{j}(I)=\lambda_{k}(I)$; (h) $\lambda_{h}(I)$; (i) $\lambda_{l}(I)$; (j) $\lambda_{m}(I)$. (b) The $\dot{\Theta}$-componentgraph of $I$. Each node of the graph is a valued connected component made of a connected component (A-F) of a thresholded image depicted in $(\mathrm{e}-\mathrm{j})$, and a value corresponding to the associated colour in (d). (c) The "compact" $\dot{\Theta}$ component-graph $\left(\dot{\Theta} / \sim_{\dot{\theta}}, \dot{4}\right)$ of $I$, which has the same structure as $(\Psi, \subseteq)$ (see Property 5).

Property 5: By considering the bijection between $\dot{\Theta} / \sim_{\dot{\theta}}$ and $\Psi$ which associates any equivalence class $[(X, v)]_{\sim_{\dot{\theta}}}$ of $\sim_{\dot{\theta}}$ to $X$,

$$
\left(\dot{\Theta} / \sim_{\dot{\theta}}, \unlhd\right) \text { is isomorphic to }(\Psi, \subseteq) .
$$

Proof: Formula (27) derives from the definitions of $\unlhd$ and $\sim_{\dot{\theta}}$

\section{Links between $(\ddot{\Theta}, \ddot{\hookrightarrow})$ and $(\dot{\Theta}, \dot{\hookrightarrow})$}

The nodes of $(\dot{\Theta}, \dot{\bullet})$ which are preserved in $(\ddot{\Theta}, \ddot{4})$ are the sup/max-generators of $I$, i.e., the valued connected components $K \in \dot{\Theta}$ which contribute effectively to the (re)construction of $I$ via their associated cylinder function $C_{K}$ (see Formulae (13) and (17)). This property can however be expressed without directly considering the relations between $I$ and the cylinder functions induced by $\dot{\Theta}$.

Property 6: Let $K=(X, v) \in \dot{\Theta}$. We have

$$
(K \in \ddot{\Theta}) \Leftrightarrow\left((K \in \stackrel{\unlhd}{\triangle} \dot{\Theta}) \vee\left(K \neq \bigsqcup \stackrel{\unlhd}{\triangle} K^{\downarrow}\right)\right)
$$

Proof: First note that $(\Omega, \perp)$ satisfies Formula (28). Let us now suppose that $K \neq(\Omega, \perp)$. If $K=(X, v) \in \Delta^{\unlhd} \dot{\Theta}$, then for all $x \in X$, we have $I(x)=v$. If $K \neq \sqcup \triangle^{-} K^{\downarrow}$, then, there exists $x \in X$ such that $I(x)=v$. The fact that $K \in \ddot{\Theta}$ then derives from Formula (13). If $K \notin \Delta^{\unlhd} \dot{\Theta}$ and $K=\sqcup \triangle^{\unlhd} K^{\downarrow}$, then for each $x \in X$, there exists $K^{\prime} \in$
$K^{\downarrow}$ such that $v<C_{K^{\prime}}(x)$. Consequently, we have $C_{K}(x)<$ $\Upsilon_{K^{\prime} \in \Delta^{\unlhd} K^{\downarrow}}^{\leq} C_{K^{\prime}}(x)$, and then, from Formula (13), $K \notin \ddot{\Theta}$.

As a corollary of this property, when $[K]_{\sim_{\dot{\theta}}} \neq\{K\}$, we necessarily have $K \notin \ddot{\Theta}$. The links between $\dot{\Theta}$ - and $\ddot{\Theta}$ component-trees are exemplified in Fig. 2(n,o).

Note finally that there is no straightforward way to locally characterise the edges of $\ddot{\Theta}$ from the ones of $\dot{\Theta}$. Indeed, the existence (resp. non-existence) of an edge between two nodes of $\ddot{\Theta}$ depends on the non-existence (resp. existence) of a transitive path composed of successive $\ddot{\Theta}$-edges between these nodes.

\section{About the influence of $(V, \leqslant)$ ON COMPONENT-GRAPHS}

In this section, we study how certain order relations $\leqslant$ defined on $V$ influence the structural properties of the component-graphs. In particular, we consider (from the most general to the most specific) three kinds of ordered sets, frequent in image applications: lower-piecewise lattices (Section VII-A), lower-piecewise totally ordered sets (Section VII-B), and totally ordered sets (Section VII-C).

\section{A. $(V, \leqslant)$ is a lower-piecewise lattice}

An ordered set $(X, \leqslant)$ is a upper- (resp. lower-)semilattice if for any $x, y \in X, \mathrm{~V}^{\leqslant}\{x, y\}$ (also noted $x \mathrm{~V}^{\leqslant} y$ ) (resp. $\Lambda^{\leqslant}\{x, y\}$ (also noted $x \wedge \leqslant y$ ) exists. It is a lattice if it is both a upper- and a lower-semilattice.

An ordered set $(X, \leqslant)$ is a upper- (resp. lower-)piecewise lattice if for any $x \in X$, the ordered set $\left(x^{\uparrow}, \leqslant\right)\left(\right.$ resp. $\left.\left(x^{\downarrow}, \leqslant\right)\right)$ is a lattice. Note that a upper- (resp. lower-)semilattice is a upper- (resp. lower-)piecewise lattice, but the converse is not true in general.

In this section, we assume that $(V, \leqslant)$ is a lower-piecewise lattice.

1) Structure of the $\Theta$-component-graphs: The $\Theta$ component-graphs inherit from the structure of $(V, \leqslant)$.

Property 7: Let $(V, \leqslant)$ be a lower-piecewise lattice. Then

$$
(\Theta, \unlhd) \text { is a upper-piecewise lattice. }
$$

Proof: Let $K=(X, v) \in \Delta^{\unlhd} \Theta$. It derives from Property 2 that $\left(K^{\uparrow}, \unlhd\right)$ is a lattice. Since for any $x \in X$ where $(X, \unlhd)$ is a lattice, $\left(x^{\uparrow}, \unlhd\right)$ is still a lattice, $(\Theta, \unlhd)$ is a upper-piecewise lattice.

As a corollary, we have the following property, related to the structure of the equivalence classes of $\sim_{\theta}$.

Property 8: Let $(V, \leqslant)$ be a lower-piecewise lattice. Let $K \in \Theta$, then

$$
\left([K]_{\sim_{\theta}}, \unlhd\right) \text { is a lower-semilattice. }
$$

Proof: Let $K=(X, v)$. Let $K^{\prime}=(Y, u) \in \Delta^{\unlhd} \Theta$ such that $Y \subseteq X$. From Property $7,\left(K^{\prime \uparrow}, \unlhd\right)$ is a lattice. Moreover we have $[K]_{\sim_{\theta}} \subseteq K^{\prime \uparrow}$. As $(V, \leqslant)$ is a lower-piecewise lattice, $\left(u^{\downarrow}, \leqslant\right)$ is a lattice. Let $\left(X, v_{1}\right),\left(X, v_{2}\right) \in[K]_{\sim_{\theta}}$. We have $X \subseteq \lambda_{v_{1} \vee \leqslant v_{2}}(I)$, and then, from Property (P3), $X \in$ $\mathcal{C}\left[\lambda_{v_{1} \vee \leqslant v_{2}}(I)\right]$. Consequently, we have $\left(X, v_{1} \vee \leqslant v_{2}\right) \in[K]_{\sim_{\theta}}$, and the result follows. 


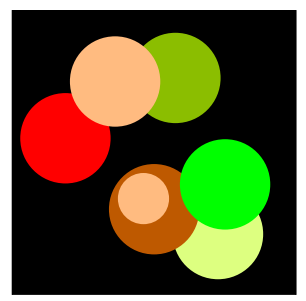

(a) $I$

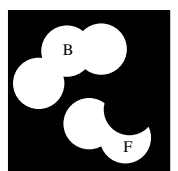

(d) $\lambda_{(0,1)}(I)$

(c) $\lambda_{(0,0)}(I)$
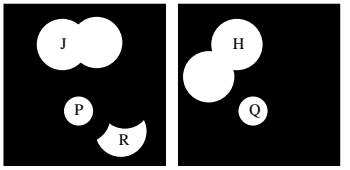

(h) $\lambda_{(2,0)}(I)$
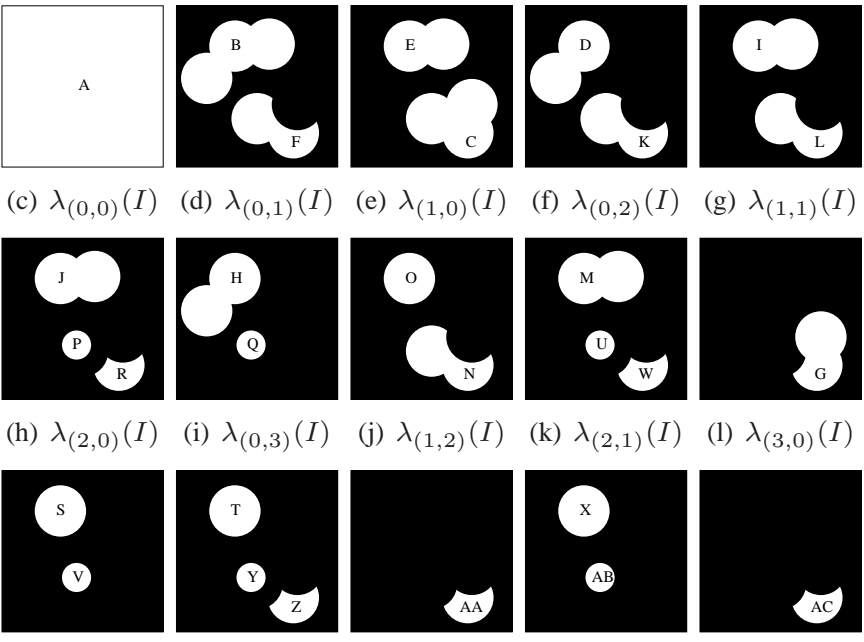

(e) $\lambda_{(1,0)}(I)$

(f) $\lambda_{(0,2)}(I)$

(g) $\lambda_{(1,1)}(I)$
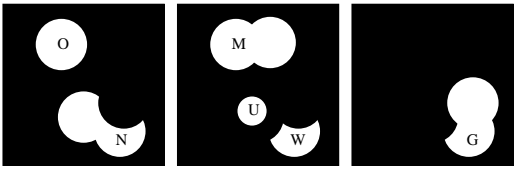

(k) $\lambda_{(2,1)}(I) \quad(1) \lambda_{(3,0)}(I)$

(m) $\lambda_{(1,3)}(I)$ (n) $\lambda_{(2,2)}(I)$ (o) $\lambda_{(3,1)}(I)$ (p) $\lambda_{(2,3)}(I)$ (q) $\lambda_{(3,2)}(I)$

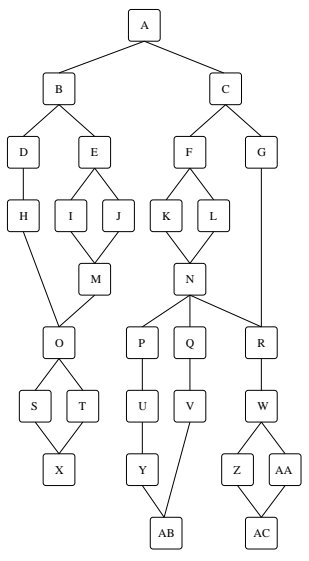

(r) $\mathfrak{G}$

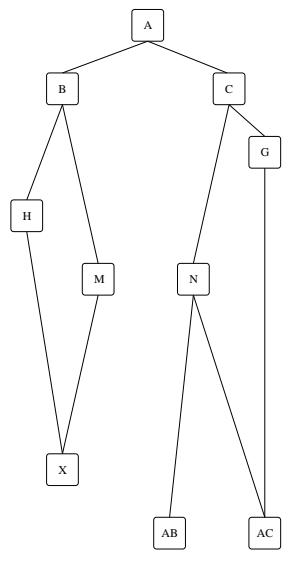

(s) $\dot{\mathfrak{G}}$
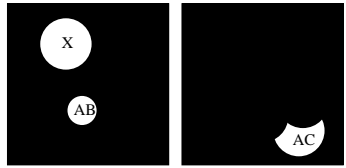

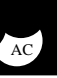

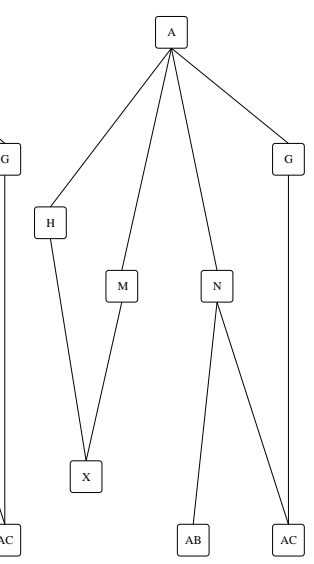

(t) $\ddot{\mathfrak{G}}$
Fig. 4. (a) A multivalued image $I: \Omega \rightarrow V$ with $\Omega \subset \mathbb{R}^{2}$, and $V \subseteq \mathbb{Z}^{2}$, equipped with the lexicographic order relation. (b) The Hasse diagram of the ordered set $(V, \leqslant)$. For the sake of readability, each value of $V$ is associated to an arbitrary colour. (c-q) Thresholded images $\lambda_{v}(I)$ for $v \in V$. (r) The $\Theta$-component-graph of $I$. The letters $(\mathrm{A}-\mathrm{AC})$ in nodes correspond to the associated connected components $(\mathrm{c}-\mathrm{q})$. (s) The $\dot{\Theta}$-component-graph of $I$. The nodes $\mathrm{H}, \mathrm{M}, \mathrm{N}$ and $\mathrm{X}$ are associated to the sets of nodes $\{\mathrm{D}, \mathrm{H}\},\{\mathrm{E}, \mathrm{I}, \mathrm{J}, \mathrm{M}\}$, $\{\mathrm{F}, \mathrm{K}, \mathrm{L}, \mathrm{N}\}$ and $\{\mathrm{O}, \mathrm{S}, \mathrm{T}, \mathrm{X}\}$ in $(\mathrm{r})$, respectively. (t) The $\ddot{\Theta}$-component-graph of $I$. The nodes $\mathrm{B}$ and $\mathrm{C}$, which were present in (s) have disappeared, since they are spatially surrounded by $\{\mathrm{H}, \mathrm{M}\}$ and $\{\mathrm{G}, \mathrm{N}\}$, respectively.

It then derives from this property that the $\dot{\Theta}$-componentgraphs also inherit from the structure of $(V, \leqslant)$.

Property 9: Let $(V, \leqslant)$ be a lower-piecewise lattice. Then

$$
(\dot{\Theta}, \unlhd) \text { is a upper-piecewise lattice. }
$$

Proof: The result derives from the fact that $(\Theta, \unlhd)$ is a upper-piecewise lattice and that $\left([K]_{\sim_{\theta}}, \unlhd\right)$ admits a minimum for any $K \in \Theta$.

Note that, in general, $(\ddot{\Theta}, \unlhd)$ is not a upper-piecewise lattice. These properties are exemplified in Fig. 4.

2) Links between $\Theta$ - and $\dot{\Theta}$-component-graphs: Under the current hypotheses (see Property 8), Formula (12) can be rewritten as

$$
\dot{\Theta}=\left\{\left(X, \bigvee_{X \in \mathcal{C}\left[\lambda_{v}(I)\right]}^{\lessgtr} v\right) \mid X \in \Psi\right\}
$$

This formula leads to the following result.

Property 10: Let $(V, \leqslant)$ be a lower-piecewise lattice. By considering the bijection between $\dot{\Theta}$ and $\Psi$ which associates any node $(X, v)$ to $X$,

$$
(\dot{\Theta}, \unlhd) \text { is isomorphic to }(\Psi, \subseteq) .
$$

Proof: From Formula (32), for each $K \in \dot{\Theta}$, we have $[K]_{\sim_{\dot{\theta}}}=\{K\}$. The result then follows from Property 5 .

Moreover, by extending the relation $\unlhd$ (and then ४) from $\Theta$ to $\Theta / \sim_{\theta}$ as follows

$$
\left(\left[K_{1}\right]_{\sim_{\theta}} \unlhd\left[K_{2}\right]_{\sim_{\theta}}\right) \Leftrightarrow\left(\stackrel{\unlhd}{\mathcal{\Lambda}}\left[K_{1}\right]_{\sim_{\theta}} \unlhd \stackrel{\unlhd}{\wedge}\left[K_{2}\right]_{\sim_{\theta}}\right)
$$

we have the following property.

Property 11: If $(V, \leqslant)$ is a lower-piecewise lattice, then

$$
\left(\Theta / \sim_{\theta}, \unlhd\right) \text { is isomorphic to }(\dot{\Theta}, \unlhd) .
$$

Proof: The proof derives from the equality between any $K \in \dot{\Theta}$ and $\boldsymbol{\Lambda}^{\unlhd}[K]_{\sim_{\theta}}$.

This identification is completed by the fact that for all $K \in \dot{\Theta}$, $\left([K]_{\sim_{\theta}}, \unlhd\right)$ is a lower-semilattice (Property (8)), and by the following property.

Property 12: Let $(V, \leqslant)$ be a lower-piecewise lattice. For all $K_{1}, K_{2} \in \Theta$ (with $\left[K_{1}\right]_{\sim_{\theta}} \neq\left[K_{2}\right]_{\sim_{\theta}}$ ) we have

$$
\left(K_{1} \triangleleft K_{2}\right) \Rightarrow\left(\stackrel{\unlhd}{\text { 人 }}\left[K_{1}\right]_{\sim_{\theta}} \triangleleft \stackrel{\unlhd}{\text { 人 }}\left[K_{2}\right]_{\sim_{\theta}}\right)
$$

Proof: The result derives from Property 4.

Broadly speaking, all the edges between two nodes of $(\Theta, \mathbf{4})$ associated to a same connected component disappear in $(\dot{\Theta}, \dot{4})$, while any edge between two nodes of $(\Theta, \mathbf{4})$ associated to distinct connected components lead to edges between the nodes of $(\dot{\Theta}, \dot{4})$ respectively associated to these two distinct connected components. These links between $\Theta$ and $\dot{\Theta}$-component-trees are exemplified in Fig. 4(r,s).

\section{B. $(V, \leqslant)$ is a lower-piecewise totally ordered set}

We say that an order relation $\leqslant$ on a set $X$ is upper(resp. lower-)piecewise total (and that $(X, \leqslant)$ is a upper- (resp. lower-)piecewise totally ordered set) if for any $x \in X,\left(x^{\uparrow}, \leqslant\right)$ (resp. $\left(x^{\downarrow}, \leqslant\right)$ ) is a totally ordered set.

In this section, we assume that $(V, \leqslant)$ is a lower-piecewise totally ordered set. Note that $(V, \leqslant)$ is then also a lowerpiecewise lattice. 


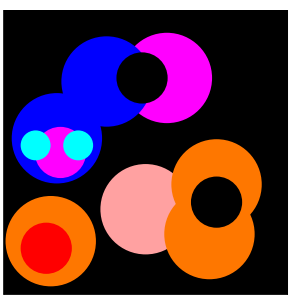

(a) $I$

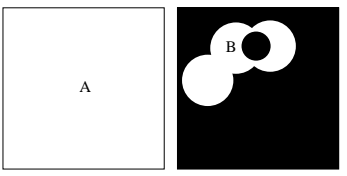

(c) $\lambda_{a}(I)$

(d) $\lambda_{b}(I)$

(e) $\lambda_{c}(I)$
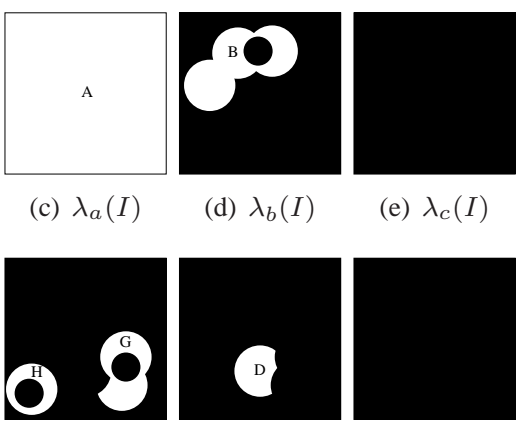

(i) $\lambda_{g}(I)$

(j) $\lambda_{h}(I)$

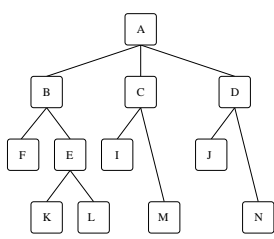

$\lambda_{i}(I)$ (m) $\mathfrak{G}$

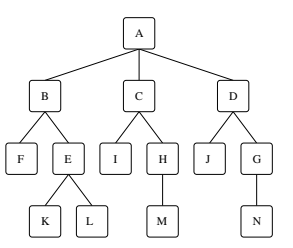

(n) $\dot{\mathfrak{G}}$

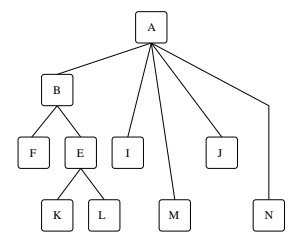

(o) $\ddot{\mathfrak{G}}$
Fig. 5. (a) A multivalued image $I: \Omega \rightarrow V$ with $\Omega \subset \mathbb{R}^{2}$, and $V=\{a, b, c, d, e, f, g, h, i, j\}$. (b) The Hasse diagram of a lower-piecewise totally ordered set $(V, \leqslant)$. For the sake of readability, each value of $V$ is associated to an arbitrary colour. (c-1) Thresholded images $\lambda_{v}(I)$ for $v \in V$. (m) The $\Theta$-component-graphs of $I$. The letters $(\mathrm{A}-\mathrm{N})$ in nodes correspond to the associated connected components (c-1). (n) The $\Theta$-component-graphs of $I$. The nodes $\mathrm{M}$ and $\mathrm{N}$ are associated, in $(\mathrm{m})$, to "linear parts" $\{\mathrm{H}, \mathrm{M}\}$ and $\{\mathrm{G}, \mathrm{N}\}$ (Properties 15 and 16). (o) The $\ddot{\Theta}$-component-graphs of $I$.

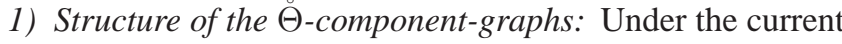
hypotheses, the result of Property 2 is strengthened.

Property 13: Let $K=(X, v) \in \Theta$. By considering the bijection $\sigma: K^{\uparrow} \rightarrow v^{\downarrow}$,

$$
\left(K^{\uparrow}, \unlhd\right) \text { is isomorphic to }\left(v^{\downarrow}, \geqslant\right) \text {. }
$$

Proof: The result derives from the totality of $\leqslant$ on $v^{\downarrow}$.

The $\Theta$-component-graphs then inherit from the structure of $(V, \leqslant)$.

Property 14: Let $(V, \leqslant)$ be a lower-piecewise totally ordered set. Then

\section{$(\Theta, \unlhd)$ is a upper-piecewise totally ordered set.}

Proof: The result derives from Property 13 and the fact that any subset of a totally ordered set is itself totally ordered.

Under the current hypotheses, the $\Theta$-component-graphs have a tree structure (of root $(\Omega, \perp$ )), as illustrated in Fig. 5(a,m-o).

2) Links between $\Theta$ - and $\dot{\Theta}$-component-graphs: Since $(V, \leqslant)$ is a lower-piecewise lattice, all the properties of Section VII-A remain valid here, and in particular Properties 10

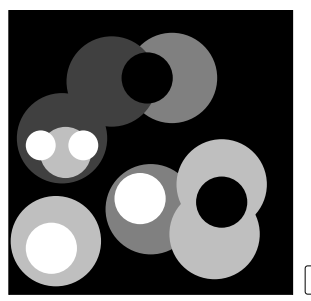

(a) $I$

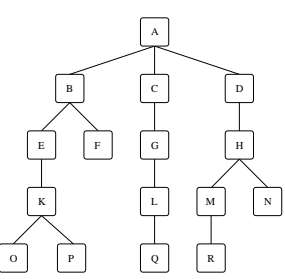

(b) $\mathfrak{G}$

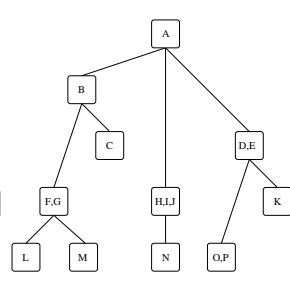

(c) $\dot{\mathfrak{G}}=\ddot{\mathfrak{G}} \sim \mathfrak{T}$

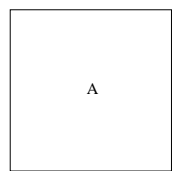

(d) $\lambda_{0}(I)$

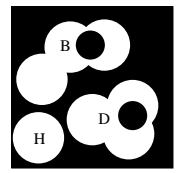

(e) $\lambda_{1}(I)$

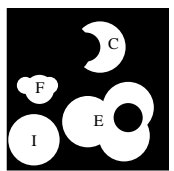

(f) $\lambda_{2}(I)$

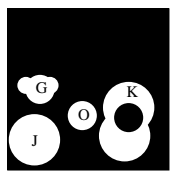

(g) $\lambda_{3}(I)$

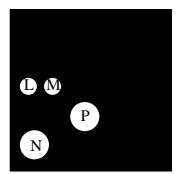

(h) $\lambda_{4}(I)$
Fig. 6. (a) The grey-level image $I: \Omega \rightarrow V$ of Fig. 1(a). (d-h) Thresholded images $\lambda_{v}(I) \subseteq \Omega$ (in white) for $v$ varying from 0 to 4 . (b) The $\Theta$ component-graph of $I$. (c) The $\dot{\Theta}$-/ $\ddot{\Theta}$-component-graphs of $I$, which are isomorphic to its component-tree (see Fig. 1(b)). The letters (A-P) in nodes correspond to the associated connected components $(\mathrm{c}-\mathrm{g})$.

and 11. Moreover, Properties 8 and 12 are strengthened by the following ones.

Property 15: Let $(V, \leqslant)$ be a lower-piecewise totally ordered set. Let $K \in \dot{\Theta}$. Then

$$
\left([K]_{\sim_{\theta}}, \unlhd\right) \text { is totally ordered. }
$$

Proof: The result derives from Property 8 and the lowerpiecewise totality of $\leqslant$.

Property 16: Let $(V, \leqslant)$ be a lower-piecewise totally ordered set. For all $K_{1}, K_{2}$ (with $\left[K_{1}\right]_{\sim_{\theta}} \neq\left[K_{2}\right]_{\sim_{\theta}}$ ) we have

$$
\begin{aligned}
& \left(K_{1} \triangleleft K_{2}\right) \Rightarrow\left(\stackrel{\unlhd}{\triangleleft}\left[K_{1}\right]_{\sim_{\theta}} \triangleleft K_{2}\right) \\
& \left(K_{1} \dot{\triangleleft} K_{2}\right) \Rightarrow\left(\stackrel{\unlhd}{\bigvee}\left[K_{1}\right]_{\sim_{\theta}} \triangleleft K_{2}\right)
\end{aligned}
$$

Proof: The result derives from Properties 12 and 15.

Broadly speaking, the branching points of the $\Theta$-componentgraph are preserved in the associated $\dot{\Theta}$-component-graph, while each node of $\dot{\Theta}$ is associated to a linear part of the $\Theta$-component-graph. These properties are illustrated in Fig. $5(\mathrm{~m}, \mathrm{n})$.

\section{C. $(V, \leqslant)$ is a totally ordered set}

In this section, we assume that $(V, \leqslant)$ is a totally ordered set. The case of grey-level images is then matched here.

Note that $(V, \leqslant)$ is also a lower-piecewise totally ordered set. Consequently, all the properties of Sections VII-A and VII-B remain valid here, and in particular Properties 10 and 14.

Moreover, Property 10 is strengthened by the following one. Property 17: Let $(V, \leqslant)$ be a totally ordered set. Then

$$
(\dot{\Theta}, \unlhd)=(\ddot{\Theta}, \unlhd)
$$

Proof: The result derives from Property 6 and Properties (P2) and (P3). 
From the following property, we finally guarantee the compliance between the concepts of component-tree and component-graphs, as illustrated in Fig. 6.

Property 18: Let $I: \Omega \rightarrow V$ be a grey-level image, and $\mathfrak{T}$ its component-tree. Then

$$
\dot{\mathfrak{G}}, \ddot{\mathfrak{G}} \text { are isomorphic to } \mathfrak{T}
$$

and

$$
I=\bigvee_{X \in \Psi}^{\leq} C_{(X, m(X))}=\bigvee_{K \in \Theta}^{\leq} C_{K}
$$

Proof: Formula (43) derives from Properties 10 and 17. Formula (44) is a rewriting of Formulae (7) and (17).

\section{SUMMARY}

\section{A. Main properties}

Table I describes the component-graphs/tree defined by the different ordered sets induced by $\Theta$ and $\Psi$. Tables II and III summarise the isomorphism relations between them, and the nature of their order. These properties are classified according to the nature of the ordered set $(V, \leqslant)$ (with the following legend in Tables I-III: TO: totally ordered; LPTO: lowerpiecewise totally ordered; LPL: lower-piecewise lattice).

TABLE I

COMPONENT-GRAPHS/TREE DEFINITIONS, ACCORDING TO $(V, \leqslant)$.

\begin{tabular}{|l|c|c|c|c|c|}
\hline$(V, \leqslant)$ & $(\Theta, \unlhd)$ & $(\dot{\Theta}, \unlhd)$ & $\left(\dot{\Theta} / \sim_{\dot{\theta}}, \unlhd\right)$ & $(\ddot{\Theta}, \unlhd)$ & $(\Psi, \subseteq)$ \\
\hline TO & $\mathfrak{G}$ & \multicolumn{3}{|c|}{$\dot{\mathfrak{G}, \mathscr{G}}$} & $\mathfrak{T}$ \\
\hline LPTO & $\mathfrak{G}$ & \multicolumn{2}{|c|}{$\dot{\mathfrak{G}}$} & $\ddot{\mathfrak{G}}$ & \\
\hline LPL & $\mathfrak{G}$ & \multicolumn{2}{|c|}{$\mathfrak{G}$} & $\mathfrak{G}$ & \\
\hline Other & $\mathfrak{G}$ & $\mathfrak{G}$ & $\mathfrak{G}$ (compact) & $\ddot{\mathfrak{G}}$ & \\
\hline
\end{tabular}

TABLE II

\begin{tabular}{|c|c|c|c|c|c|}
\hline$(V, \leqslant)$ & $(\Theta, \unlhd)$ & $(\Theta, \unlhd)$ & $\left(\Theta / \sim_{\dot{\theta}}, \unlhd\right)$ & $(\Psi, \subseteq)$ & $(\Theta, \unlhd)$ \\
\hline TO & & \multicolumn{4}{|c|}{ Isomorphic } \\
\hline LPTO & & \multicolumn{3}{|c|}{ Isomorphic } & \\
\hline LPL & & \multicolumn{3}{|c|}{ Isomorphic } & \\
\hline Other & & \multicolumn{3}{|c|}{ Isomorphic } & \\
\hline
\end{tabular}

ISOMORPHISM RELATIONS, ACCORDING TO $(V, \leqslant)$.

TABLE III

\begin{tabular}{|c|c|c|c|c|c|}
\hline$(V, \leqslant)$ & $(\Theta, \unlhd)$ & $(\Theta, \unlhd)$ & $\left(\Theta / \sim_{\dot{\theta}}, \unlhd\right)$ & $(\Psi, \subseteq)$ & $(\Theta, \unlhd)$ \\
\hline TO & \multicolumn{5}{|c|}{ Upper-piecewise totally ordered } \\
\hline LPTO & \multicolumn{5}{|c|}{ Upper-piecewise totally ordered } \\
\hline LPL & \multicolumn{4}{|c|}{ Upper-piecewise lattice } & \\
\hline Other & & & & & \\
\hline
\end{tabular}

NATURE OF THE ORDERED SETS, ACCORDING TO $(V, \leqslant)$.

\section{B. Space complexity}

From an algorithmic point of view (and, a fortiori, from an

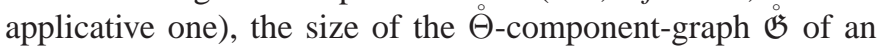
image $I: \Omega \rightarrow V$ strongly conditions the ability to process this image via a filtering framework relying on $\mathfrak{G}$. From a theoretical point of view, we have the following upper-bounds for the number of nodes of $\mathfrak{G}$

$$
\begin{aligned}
|\Theta| & =\mathcal{O}(|\Phi| \cdot|V|) \\
|\dot{\Theta}| & =\mathcal{O}(|\Phi| \cdot|V|) \\
|\ddot{\Theta}| & =\mathcal{O}(|\Phi|)
\end{aligned}
$$

where $\Phi \subseteq 2^{\Omega}$ is the set of all the flat zones of $I$, with thus $|\Phi| \leq|\Omega|$ (and generally, $|\Phi| \ll|\Omega|$ ). From a practical point of view, for real images, the actual number of nodes in the $\Theta$-component-graph of an image $I: \Omega \rightarrow V$ will be generally (much) lower than these bounds, since it will depend on $|\Phi|,|V|$, but also on the structure of the image itself, and in particular the number of its maxima and their "height", with respect to $(V, \leqslant)$. In particular, we will have

$$
|\ddot{\Theta}| \leq|\dot{\Theta}| \leq|\Theta| \leq \sum_{K \in \Delta^{\unlhd} \Theta}\left|\sigma(K)^{\downarrow}\right|
$$

\section{CONCLUSION}

The notion of component-graph has been proposed as an extension of the notion of component-tree for images taking their values in any (partially or totally) ordered sets. Some structural properties of three variants of component-graphs have been investigated, in particular for value spaces which are likely to appear in real images (e.g., piecewise totally ordered sets or lattices).

In the next part of this work, we will propose to generalise the standard grey-level image filtering framework based on component-trees [8], [14], thus relaxing the constraints linked to total orderings on image values. In particular, the component-graphs(s) construction and the non-trivial issue of image reconstruction will be discussed.

\section{REFERENCES}

[1] B. Naegel and N. Passat, "Component-trees and multivalued images: A comparative study," in Proc. ISMM, 2009, pp. 261-271.

[2] N. Passat and B. Naegel, "An extension of component-trees to partial orders," in Proc. ICIP, 2009, pp. 3981-3984.

[3] D. Wishart, "Mode analysis: A generalization of the nearest neighbor," in Numerical Taxonomy. Academic Press, 1969, pp. 282-319.

[4] J. A. Hartigan, "Statistical theory in clustering," J. Classif., vol. 2, no. 1, pp. 63-76, 1985.

[5] P. Hanusse and P. Guillataud, "Sémantique des images par analyse dendronique," in Proc. RFIA, 1991, pp. 577-588.

[6] L. Chen, M. W. Berry, and W. W. Hargrove, "Using dendronal signatures for feature extraction and retrieval," Int. J. of Imag. Syst. Tech., vol. 11, no. 4, pp. 243-253, 2000.

[7] J. Mattes and J. Demongeot, "Efficient algorithms to implement the confinement tree," in Proc. DGCI, 2000, pp. 392-405.

[8] P. Salembier, A. Oliveras, and L. Garrido, "Anti-extensive connected operators for image and sequence processing," IEEE Trans. Image Proc., vol. 7, no. 4, pp. 555-570, 1998.

[9] L. Najman and H. Talbot, Eds., Mathematical Morphology: From Theory to Applications. ISTE/J. Wiley \& Sons, 2010.

[10] P. Salembier and M. H. F. Wilkinson, "Connected operators: A review of region-based morphological image processing techniques," IEEE Sig. Proc. Magazine, vol. 26, no. 6, pp. 136-157, 2009.

[11] E. J. Breen and R. Jones, "Attribute openings, thinnings, and granulometries," Comp. Vis. Imag. Under., vol. 64, no. 3, pp. 377-389, 1996.

[12] L. Najman and M. Couprie, "Building the component tree in quasi-linear time," IEEE Trans. Image Proc., vol. 15, no. 11, pp. 3531-3539, 2006.

[13] M. H. F. Wilkinson, H. Gao, W. H. Hesselink, J.-E. Jonker, and A. Meijster, "Concurrent computation of attribute filters on shared memory parallel machines," IEEE Trans. Pattern Anal. Mach. Intell., vol. 30, no. 10, pp. 1800-1813, 2008. 
[14] R. Jones, "Connected filtering and segmentation using component trees," Comp. Vis. Imag. Under., vol. 75, no. 3, pp. 215-228, 1999.

[15] P. Dokládal, I. Bloch, M. Couprie, D. Ruijters, R. Urtasun, and L. Garnero, "Topologically controlled segmentation of 3D magnetic resonance images of the head by using morphological operators," Pattern Recogn., vol. 36, no. 10, pp. 2463-2478, 2003.

[16] G. K. Ouzounis and M. H. F. Wilkinson, "Mask-based second-generation connectivity and attribute filters," IEEE Trans. Pattern Anal. Mach. Intell., vol. 29, no. 6, pp. 990-1004, 2007.

[17] N. Passat, B. Naegel, F. Rousseau, M. Koob, and J.-L. Dietemann, "Interactive segmentation based on component-trees," Pattern Recogn., vol. 44, no. 10-11, pp. 2539-2554, 2011.

[18] J. Mattes, M. Richard, and J. Demongeot, "Tree representation for image matching and object recognition," in Proc. DGCI, 1999, pp. 298-312.

[19] V. Mosorov, "A main stem concept for image matching," Pattern Recognit. Lett., vol. 26, no. 8, pp. 1105-1117, 2005.

[20] N. Alajlan, M. S. Kamel, and G. H. Freeman, "Geometry-based image retrieval in binary image databases," IEEE Trans. Pattern Anal. Mach. Intell., vol. 30, no. 6, pp. 1003-1013, 2008.

[21] E. R. Urbach, J. B. T. M. Roerdink, and M. H. F. Wilkinson, "Connected shape-size pattern spectra for rotation and scale-invariant classification of gray-scale images," IEEE Trans. Pattern Anal. Mach. Intell., vol. 29, no. 2, pp. 272-285, 2007.

[22] M. A. Westenberg, J. B. T. M. Roerdink, and M. H. F. Wilkinson, "Volumetric attribute filtering and interactive visualization using the max-tree representation," IEEE Trans. Image Proc., vol. 16, no. 12, pp. 2943-2952, 2007.

[23] D. Menotti, L. Najman, and A. de Albuquerque Araújo, "1D component tree in linear time and space and its application to gray-level image multithresholding," in Proc. ISMM, 2007, pp. 437-448.

[24] B. Naegel and L. Wendling, "A document binarization method based on connected operators," Pattern Recognit. Lett., vol. 31, no. 11, pp. 1251-1259, 2010.

[25] E. R. Urbach, N. J. Boersma, and M. H. F. Wilkinson, "Vector attribute filters," in Proc. ISMM, 2005, pp. 95-104.

[26] E. Aptoula and S. Lefèvre, "A comparative study on multivariate mathematical morphology," Pattern Recogn., vol. 40, no. 11, pp. 2914 2929, 2007.

[27] C. Ronse and V. Agnus, "Morphology on label images: Flat-type operators and connections," J. Math. Imaging Vis., vol. 22, no. 2, pp 283-307, 2005.

[28] V. Barnett, "The ordering of multivariate data," J. Roy. Stat. Soc. A Sta., vol. 139 , no. 3, pp. 318-354, 1976.

[29] J. Goutsias, H. J. A. M. Heijmans, and K. Sivakumar, "Morphological operators for image sequences," Comp. Vis. Imag. Under., vol. 62, no. 3, pp. 326-346, 1995.

[30] J. Angulo, "Morphological colour operators in totally ordered lattices based on distances: Application to image filtering, enhancement and analysis," Comp. Vis. Imag. Under., vol. 107, no. 1-2, pp. 56-73, 2007.

[31] J. Angulo and J. Serra, "Modelling and segmentation of colour images in polar representations," Image Vision Comput., vol. 25, no. 4, pp. 475495, 2007.

[32] J. Angulo, "Geometric algebra colour image representations and derived total orderings for morphological operators-Part I: Colour quaternions," J. of Vis. Commun. Image R., vol. 21, no. 1, pp. 33-48, 2010.

[33] D. Gimenez and A. N. Evans, "An evaluation of area morphology scalespaces for colour images," Comp. Vis. Imag. Under., vol. 110, no. 1, pp. 32-42, 2008.

[34] P. Soille, "Constrained connectivity for hierarchical image partitioning and simplification," IEEE Trans. Pattern Anal. Mach. Intell., vol. 30, no. 7, pp. 1132-1145, 2008.

[35] L. Mazo, N. Passat, M. Couprie, and C. Ronse, "Paths, homotopy and reduction in digital images," Acta Appl. Math., vol. 113, no. 2, pp. 167193, 2011.

[36] C. R. F. Maunder, Algebraic Topology. Dover, 1996.

[37] R. E. Stong, "Finite topological spaces," T. Am. Math. Soc., vol. 123, no. 25, pp. 325-340, 1966.

[38] A. Rosenfeld, "Connectivity in digital pictures," J. Assoc. Comput. Mach., vol. 17, no. 1, pp. 146-160, 1970.

[39] T. Y. Kong and A. Rosenfeld, "Digital topology: Introduction and survey," Comput. Vision Graph., vol. 48, no. 3, pp. 357-393, 1989.

[40] V. A. Kovalevsky, "Finite topology as applied to image analysis," Comput. Vision Graph., vol. 46, no. 2, pp. 141-161, 1989.

[41] C. Ronse, "Set-theoretical algebraic approaches to connectivity in continuous or digital spaces," J. Math. Imaging Vis., vol. 8, no. 1, pp. 41-58, 1998.
[42] J. Serra, "Connectivity on complete lattices," J. Math. Imaging Vis., vol. 9, no. 3, pp. 231-251, 1998 .

[43] U. Braga-Neto and J. Goutsias, "Connectivity on complete lattices: New results," Comp. Vis. Imag. Under., vol. 85, no. 1, pp. 22-53, 2002. 